\title{
35. STELLAR CONSTITUTION
}

President: P.Demarque (USA)

Vice-President: C.Chiosi (Italy)

Members: R. Canal, I. Iben, A. Maeder (past President),

G. Michaud, K. Nomoto, A. Renzini, A.V. Tutukov,

D. VandenBerg, G. Vauclair

\section{INTRODUCTION}

This report covers a few highlights of the research carried out during the last triennium. It focusses nearly exclusively on areas which have been touched by the recent developments in the equation of state and opacities of stellar matter. These developments, coupled with the remarkable and accelerating progress on internal dynamics and transport processes in the interiors of stars, have literally transformed the field in a very short time.

The new generation of stellar opacities and equation of state was a highlight of the IAU XXI General Assembly in Buenos Aires. Since then, these opacities, particularly the OPAL interior opacities due to Iglesias \& Rogers (ApJ,371,408) which have been the most accessible to the community, have been used with remarkable success on a number of problems. One must also emphasize the equally important ongoing work on low temperature opacities which include the effects of molecules (Sharp A\&AS,94,1 and Kurucz 1991). The task is still unfinished in the low temperature range, but it will have a profound impact on our understanding of stellar interiors.

The topics briefly covered in this report are: convection in stars, the physics of the interior of the Sun and sun-like stars, including pulsation, rotation and diffusion, and the evolution of low mass and massive stars.

Books published:

Solar Interior and Atmosphere, A.N. Cox , W.C. Livingston and M.S. Matthews, eds, University of Arizona Press, Tucson,1991

Challenges to theories of the structure of moderate-mass stars, Lecture Notes Phys. Vol.388, D.Gough \& J.Toomre,eds.,Springer,Berlin,1991

The Sun, an introduction, by M.Stix,Springer,Berlin 
The Physics of Astrophysics, Vol.I: Radiation and Vol.II: Gas Dynamics, F.H.Shu, Univ.Sci.Books,Mill Valley,CA

Proceedings of meetings:

Proc. IAU Symp. 155, Planetary nebulae, R.Weinberger \& A.Acker.eds., Kluwer, Dordrecht, 1992

Proc. IAU Symp. 149, The stellar populations of galaxies, B.Barbuy \& A.Renzini,eds., Kluwer, Dordrecht, 1992

Proc. IAU Symp. 151, Processes in interacting binaries, Y.Kondo, R.F.Sistero, R.S.Polidan, eds., Kluwer, Dordrecht, 1992

Proc. IAU Colloq. 137, Inside the stars, ASP conf.ser. Vol.40, W.W.Weiss \& A. Baglin, eds., 1993

Proc. Cool stars, stellar systems and the Sun.7., ASP conf.ser. Vol.26, M.S.Giampapa \& J.A.Bookbinder, eds., 1992

Vulcano workshop on Young star clusters and early stellar evolution, F.Palla, P.Persi, H.Zinnecker, eds., Mem.Soc.Astron.Ital.,62,705,1991

Workshop on astrophysical opacities, A.E.Lynes-Gray, C.Mendoza, C.J.Zeippen, eds.,Rev.Mex.Astron.Astrof.,Vol.23,special issue, 1992

Teramo Workshop on star clusters and stellar evolution, E.Brocato, F.R.Ferraro, G.Piotto, eds.,Mem.Soc.Astron.Ital.,63,1,1992

Proc. intern. symp. on origin and evolution of the elements, Tokyo, S.Kübono, T.Kajino, eds., World Scientific, 1993

Articles in Annual Reviews of Astronomy \& Astrophysics

Vol.29, 1991 Chemical evolution of the Galaxy, by N.C. Rana, p.129

Radioactive dating of the elements, by J.J.Cowan, F.-K. Thielemann, J.W. Truran, p.447

Seismic observations of the Solar Interior, by D.Gough and J.Toomre, p.627

Vol.30, 1992 Magnetic fields of degenerate stars, by G. Chanmugan, p.143

New developments in understanding the HR diagram, by C.Chiosi, G.Bertelli, A.Bressan, p.235

Evidence for black holes in stellar binary systems, by A.P.Cowley, p.287 
Type Ia Supernovae as standard candles, by D.Branch, G.A.Tammann, p.359

Vol.31, 1993 Origin and evolution of $\mathrm{X}$-ray binaries and binary radio pulsars, by F.Verbunt, p.93

\section{CONVECTION IN STARS}

\subsection{Stellar convection zones}

Important progress has been made in stellar convection, both in simulating numerically stellar compressible convection, and in introducing more realistic treatments of convection to stellar evolution calculations. New efforts are also aimed at including a description of the effects of magnetic fields in some of the models.

\subsection{Numerical simulations of convection}

One of the outstanding unsolved problems in calculations of stellar structure and evolution has been the treatment of convection in stars. It has recently become possible to perform increasingly realistic simulations of compressible convection in two and three dimensions (Chan \& Sofia ApJ,336,1022; Cattaneo et al. ApJ,370,282; Chan \& Serigawa 54.065.035; Stein \& Nordlund,ApJ,342,L95; Hossain \& Mullen Ap.J,380,631; Rast et al. ApJ,408,L53). Under some circumstances and provided they include an' adequate treatment of radiative effects near the surface, these models can in principle be tested agaist observable stellar features such as granulation and effects on spectral lines (Kim \& Fox,in Cool Stars,Stellar Systems \& the Sun.7.,p.172).

\subsection{New descriptions of convection in stellar models}

One of the goals of these numerical simulations has been to replace the mixing length theory (MLT) formulation of convection, universally adopted in stellar structure calculations, by a physically more realistic description, and one that is free of the arbitrariness associated with the mixing-length parameter.

\subsubsection{A parameterization of convection simulations}

The first successful attempt has now been made by Lydon, Fox \& Sofia (ApJ,397,701;ApJ,403,L79) to construct an evolutionary model of the solar interior which matches the solar radius without recourse to any adjustable mixing length parameter to describe the convection zone. The formulation of convection is based on the three-dimensional simulations of compressible, stratified convection of Chan \& Sofia (ApJ,336,1022). Other physical assumptions in the models are fully consistent with the recent state-of-the-art standard models of the Sun described in the next section. The same formulation was subsequently applied by Lydon et al. (ApJ,1993) to the 
binary system Alpha Centauri whose two members are solar analogues with masses which bracket the mass of the Sun. They discussed their models within the context of the known uncertainties in the observed parameters of Alpha Centauri. Other recent evolutionary models for the two components of Alpha Centauri were constructed by Noels et al.(A\&A,247,91) and by Edmonds et al.(ApJ,394,313). The latter also calculated the p-mode nonradial oscillation spectrum for both stars.

\subsubsection{The Canuto-Mazzitelli model of convection}

Another attempt to free the representation of stellar convection from the adjustable mixing length has been made by Canuto (ApJ,392,218) and Canuto \& Mazzitelli (ApJ,370,295). These authors' approach is based on a theoretical model of turbulence, analogous to terrestrial atmospheric turbulence. In order to model the effects of compressibility, the scale of the convective region is used so that the mixing length is removed as an explicit parameter in the equations. The formulation has been applied to the Sun. The $\mathrm{p}$-mode spectrum of the solar models has been investigated by Paterno et al.(ApJ,402,733). An attempt to reproduce theoretically the color-magnitude diagram of the old open star cluster M67 has been made by D'Antona et al.(A\&A,257,539).

\subsection{Magnetic convection}

The problems of magnetic convection have recently been considered by Hanami \& Tajima (Ap.J,377,694), Fox et al. (ApJ,383,860), Cattaneo \& vainshtein (ApJ,376,L21), Fisher et al. (Apj,374,766) and Nordlund et al. (ApJ,392,747).

\section{THE SOLAR INTERIOR, HELIOSEISMOLOGY AND SOLAR NEUTRINOS}

The last few years have been particularly active in the area of solar modelling. Significant advances have been largely stimulated by helioseismology and neutrino experiments, and facilitated by spectacular advances in opacity calculations and in improvements in the calculation of the equation of state.

\subsection{Helioseismology}

Important advances:

3.1.1. The depth of the solar convection zone has been evaluated with precision by inversion of the p-mode data by Christensen-Dalsgaard, Gough and Thompson (ApJ,378,413).

3.1.2. Improved equation of state and OPAL opacities in the solar interior (Iglesias \& Rogers ApJ,371,408;ApJL;371,L73) lead to agreement with observed p-mode spectrum, within the errors of the physics for the first time in standard models of the Sun. The sensitivity of the p-mode frequencies to 
the known errors in the solar age, its chemical composition, the equation of state, the atmosphere structure, the boundary conditions, the shell resolution of the model, and the nuclear reaction rates have been explored systematically (Cox, Guzik and Raby ApJ,353; Faulkner \& Swenson ApJ,386,L55; Guenther et al. ApJ,387,372; Berthomieu et al. CESAM models A\&A,268,775). A similar analysis was also performed for g-modes (Guenther et al.ApJ,392,328). Non-adiabatic effects on the p-mode spectrum have been explored by Balmforth (MNRAS,255,603;255,632;255,639), Cox and Guzik (1993,ApJ in press) and Guenther (1993,ApJ in press).

3.1.3. Concern with the effects of the diffusion of helium on the solar pmode frequencies which were found to be affected at the same level as the current uncertainties in the other input physics. Detailed studies of helium diffusion in the Sun have been made by Guzik \& Cox (ApJ,386,729); Goode et al. (ApJ,387,707); Dziembowski et al. (MNRAS,249,602); Kosovichev et al.(1993,MNRAS in press); Christensen-Dalsgaard et al.(ApJ,403,L75); Proffitt \& Thompson (1993 ApJ in press); Guenther, Pinsonneault \& Bahcall (1993,ApJ in press). For a comparison of diffusion coefficients used in stellar interior calculations, see also Chaboyer et al.(ApJ,388,372).

\subsection{Solar neutrinos}

The main improvements have been:

3.2.1. Upgraded energy generation rates by Bahcall \& Pinsonneault (ApJ,); but see also the discussion of Turck-Chieze \& Lopes (ApJ,408,347).

3.2.2. The inclusion of helium diffusion and non-adiabatic effects in the pulsation of the models (Guenther et al.1993, ApJ in press).

3.2.3. Comparisons with the preliminary gallium experimental results.

\subsection{Seismology of solar analogues}

The seismology of the evolving Sun has been considered by Guenther (ApJ,375,352). Edmonds et al. (ApJ,394,313) have explored the evolutionary status and seismology of solar analogues Alpha Centauri A \& B. Guenther \& Demarque (ApJ,405,298) have studied the evolutionary status and seismology of Procyon A. Other recent highlights of solar interior physics are discussed in the sections on convection and the solar-stellar connection.

\subsection{Solar rotation curve by inversion}

A great deal of effort continues to be concentrated on problems of the inversion of seismic data from the Sun, primarily for the purpose of deriving the state of rotation in the solar interior using the rotational splittings of $\mathrm{p}$ modes (e.g.Schou et al. ApJ,385,L59). 
A wealth of information on all aspects of solar interior physics and solar evolution can be found in the new compendium: Solar Interior and Atmosphere A.N. Cox , W.C. Livingston and M.S. Matthews, editors, University of Arizona Press, Tucson, 1991

\section{THE SOLAR-STELLAR CONNECTION: ROTATION, DIFFUSION, AND LITHIUM DEPLETION}

\subsection{Lithium depletion by rotation}

The depletion of lithium in the envelopes of the Sun and late-type stars of solar metallicity is a long standing problem which continues to attract much attention. Standard stellar models do not explain the observed depletion of lithium in the Sun and similar disk stars. The observations seem to be best explained by introducing a source of slow internal mixing, and many investigators believe that furbulent motions driven by rotationally induced instabilities are responsible for this mixing. Evolutionary models for rotating stars which include an explicit local description of rotational instabilities and relate the outward transfer of angular momentum from the star to internal mixing have been successful in explaining many of the trends in lithium abundances and surface rotational velocities observed in open star clusters (Pinsonneault et al. ApJ,367,239;ApJS,78,179; Thorburn et al. ApJ,415,150). A different approach to rotational depletion has been proposed by Charbonnel et al.(A\&A,255,191). Other explanations as well, such as mass loss, have been advanced to explain the lithium observations (Boothroyd et al. ApJ,377,318; Dearborn et al. ApJ,394,L61; Swenson \& Faulkner ApJ,395,654).

\subsection{Angular momentum transfer and mixing}

Theoretical discussions of the problems associated with angular momentum transfer in stars and its relation to element mixing are found in recent papers by Chaboyer \& Zahn (A\&A,353,173); Charbonneau (A\&A 259,134); MacGregor \& Brenner (ApJ,376,204); MacGregor \& Charbonneau (ApJ,397,L63); Spiegel \& Zahn (A\&A,265,106) and Zahn (A\&A,265,115). Other recent theoretical studies of stellar rotation include those by Clement (ApJ,406,651) and Durney (ApJ,407,367). Lithium dilution in F stars has been considered by Garcia \& Spruit $(54.065 .047)$, Charbonneau \& Michaud (ApJ,370,693) and Richer et al. (ApJS,82,329).

\subsection{Lithium in halo dwarf stars}

The problem of lithium depletion in halo dwarfs has special significance in the context of primordial abundances and cosmology. It is however even more difficult to study the rotational evolution of halo dwarfs because there are no young metal poor star clusters to guide theory in understanding the process of lithium depletion and its relation to rotation. Here, investigators must assume that the physical principles which model successfully disk stars can be equally applied to the stars in the halo which are very similar to disk 
stars, although they differ from them in two important respects, lower interior opacities and lower masses (Deliyannis et al.ApJ,414,740).

\subsection{Helium diffusion}

Diffusion can also affect the surface abundances of both lithium and helium in cool stars. Since halo dwarfs are too cool to exhibit helium lines, helium abundances cannot be estimated directly in these stars. But the abundance of lithium can be measured, and since both elements are expected to diffuse at nearly the same rate, lithium can be used as a proxy. Much effort has been extended in the last few years to understand the influence of helium diffusion on the structure and evolution of the Sun and sun-like stars. The effects of diffusion are expected to be most significant in metal poor halo stars. In these objects, which have the thinnest convection zones, an appreciable amount of helium could be drained from the convective envelope. This process is particularly effective when the convection zone becomes very shallow, as is the case for halo dwarf stars near the main sequence turnoff. The resulting hydrogen enrichment of the envelope could cause the star to turn toward the giant branch sooner in its evolution and thus lower age estimates of globular clusters. Some downward diffusion of helium near the center could also accelerate the exhaustion of the core in hydrogen. Calculations to estimate the effects of helium diffusion on the evolution of globular cluster stars have been carried out by Deliyannis \& Demarque (ApJ,370,L89), Proffitt \& Michaud (ApJ,371,584), Proffitt \& VandenBerg (ApJS,77,473) and Chaboyer et al. (ApJ,388,372;ApJ,394,515). But rotational instabilities induce turbulence, which can inhibit diffusion in stars. This problem has recently been treated in some detail by Chaboyer in his doctoral dissertation (Yale University 1993), who considered evolutionary sequences of rotating stars which include molecular diffusion as well.

\section{STELLAR EVOLUTION: LOW MASS STARS}

\subsection{Isochrones for halo and disk stars}

New sets of evolutionary tracks for halo stars near the main sequence turnoff have been published, some including the effects of oxygen enrichment (VandenBerg ApJ,391,685;VandenBerg \& Bergbusch ApJS,81,163), others oxygen and alpha capture element enrichment, as well as the effects of helium diffusion, both in the core and in the envelope (Chaboyer et al. ApJ,388,372;ApJ,394,515). They have been used to derive age differences and absolute ages for the globular clusters (see also Straniero \& Chieffi ApJS,76,525). Metal-rich theoretical isochrones have also been published which include revisions of the chronology of disk star clusters in the Galaxy (Castellani et al. ApJS,78,517; Demarque,P. et al. AJ,103,151; Meynet et al. A\&AS,98,477). Advanced phases of evolution are described by Lattanzio (ApJS,76,215) and Castellani \& Castellani (ApJ,407,649).

\subsection{Horizontal branch and post-horizontal branch evolution}


5.2.1 Horizontal branch (HB) evolution Interest in HB evolution has increased in the last few years, stimulated by several factors: ultraviolet observations with IUE and now with HST; renewed discussions of the nature of the second parameter of $\mathrm{HB}$ morphology, and prospects of direct $\mathrm{HB}$ observations in more distant extragalactic objects such as M31; and finally the importance of understanding the evolutionary status of RR Lyrae variables and the dependence of their absolute magnitudes on metallicity. The long standing problem of the mass discrepancy between the masses derived for double mode RR Lyrae variables (pulsation masses) and evolutionary masses seems to have been resolved, basically as a result of the OPAL opacity increase, which has changed the predicted pulsation masses.

Recent studies of HB models address primarily the effects of oxygen or alpha element enhancement, and the "core breathing" phenomenon as the star approaches central helium exhaustion (Lee ApJ,373,L43; Castellani et al. ApJS,76,911; Dorman et al. ApJ,366,115;ApJS,80,701;ApJ,409,387; Caloi \& Mazitelli A\&A,271,139).

Concerning the RR Lyrae variables, Lee (ApJ,373,L43) considers the variables in Omega Centauri. Opacity effects on the pulsation calculations are estimated by Simon \& Cox (ApJ,376,717), Cox (ApJ,381,L71), and Kovacz et al. (A\&A,259,L46). Pulsation masses in M15 are discussed by Simon (ApJ,387,162). Evolutionary masses are calculated with OPAL opacities by Yi et al.(ApJ,411,L25).

\subsubsection{Post-HB evolution}

At the same time, post-HB evolution has received particular attention. The main focus is to gain a better understanding of the masses and composition parameters which determine how much time will be spent during post-HB evolution on the very red asymptotic giant branch and/or in luminous blue phases of evolution. Such a knowledge is essential for understanding the integrated spectral energy distribution of old stellar populations. It seems that a satisfactory explanation of the ultra-violet upturn in elliptical galaxies in terms of the advanced phases of evolution of low-mass metal rich stars is at hand (Horch et al. ApJ,388,L53; Castellani et al. ApJ,389,227).

\section{STELLAR EVOLUTION: MASSIVE STARS}

\subsection{Convective core overshoot}

Interest has continued to focus on the evolution of massive stars; in particular on the importance of convective core overshoot on their evolution near the main sequence and in the core helium burning and more advanced phases of evolution (Roxburgh,I.A\&A,266,291; Brocato, Castellani A\&A,258,397). Stothers \& Chin are able to put some stringent limits on the extent of core overshoot in massive and intermediate mass stars (ApJ,381,L67;ApJ,383,820;ApJS,77,289;ApJ,390,136). They conclude that only a small amount of overshoot (less than 0.2 pressure scale height beyond the formal core boundary) is needed to match the observations when 
the OPAL opacities are used.

\subsection{Evolution and pulsation}

Other recent massive star studies include the work of Raiteri et al. (ApJ,367,228), Lamers et al. (ApJ,368,538), Brocato et al. (ApJ,410,99) and Spruit (A\&A,253,131). The pulsational instability of OB star models constructed with the OPAL opacities has been investigated by Cox et al. (ApJ,393,272).

\subsection{Apsidal motion test}

Claret \& Gimenez (A\&A,244,319) have explored the effect of convective overshoot and choice of opacities on the apsidal motion constant of massive stars. They achieve an improvement with the available observations. 